\title{
Isolation and Characterization of Mesenchymal Stem Cells from Human Liposuction Aspirate
}

\author{
Alaa Eldin S. Abdelhamid', Mohamed S. El Shahedy², Mona H. Mohammed', \\ Ghada S. Aly ${ }^{3 *}$, Bassma M. Dessouki ${ }^{3}$ \\ ${ }^{1}$ Department of Clinical Pathology, Faculty of Medicine, Suez Canal University, ${ }^{2}$ Department of Virology, Faculty \\ of Veterinarian Medicine, Suez Canal University, ${ }^{3}$ Department of Anatomy and Embryology, Faculty of Medicine, \\ Suez Canal University.
}

\begin{abstract}
Background: Adipose derived stem cells (ADSCs) are a new type of mesenchymal stem cells (MSC) resemble bone marrow stem cells in their biologic activities. DScs is multipotent stem cells can differentiate into multiple cell types including osteoblasts, chondrocytes, adipocytes, myocytes, vascular endothelial cells, and neurons. Stromal vascular fraction (SVF) corresponds to ADSC and describes the cells obtained immediately from adipose tissues after the digestion of collagenase. Adipose tissue is a promising source of adult mesenchymal stem cells for therapeutic applications because it is available in large amounts ( $100 \mathrm{ml}$ up to 1 liter) through liposuction and with minimal morbidity. Aim: To isolate and characterize the adipose derived stem cells from human liposuction aspirate. Material and Methods: liposuction aspirate was used for extraction of stromal vascular fragment (SVF). SVF were used for culturing and isolation of hADSCs. ADSCs were cultured in tissue-culture flasks using collagenase digestion method. Flow cytometric analysis was used to identify ADSCs markers using fluorescence-activated cell sorting (FACS) against CD44, CD90 and CD45. Results: Human ADSCs were isolated successfully using collagenase digestion method. After 4 days the cells appeared to be spindle in shape and formed symmetric colonies. After 7 days, the cell sheet formed $60 \%$ confluence and completed to $90 \%$ confluence after 10 days incubation. FACS analysis of ADSCs surface markers revealed expression of $\mathrm{CD}_{44}$, and $\mathrm{CD} 90$ markers in most of the adherent cells. In contrast, most adherent cells were negative for CD45. Conclusion: Human ADSCs were isolated successfully from human lipoaspirate.
\end{abstract}

Keywords: ADSCs; MSC; SVF

\section{Introduction}

Mesenchymal stem cells (MSC) is adult stem cells isolated from bone marrow, umbilical cord, epithelium and adipose tissues and receiving more attention in pre- clinical and clinical studies about cell therapy ${ }^{(1)}$. MSCs are considered good candidates for cellular therapy due to the following criteria: a) they are easily harvested; b) they can be harvested from the patient himself $c$ ) possibility of harvesting an

*Corresponding Author: dranatomy103@yahoo.com 
adequate number of cells for transplantation, due to the high cellular proliferation in vitro, d) multipotent capacity of cell differentiation and self-renewal, e) easy laboratory handling, f) they have little immunogenicity, and g) they have the ability to integrate into the host tissue and interact with the surrounding tissue. Adipose derived stem cells (ADSCs) are a new type of MSC resemble bone marrow stem cells in their biologic activities and used as alternatives for bone marrow transplantation (BMT) $)^{(2), A D S C s}$ is multipotent stem cells can differentiate into multiple cell types including osteoblasts, chondrocytes, adipocytes, myocytes, vascular endothelial cells and neurons(3). Adipose tissue is highly complex and is constituted by mesenchymal cells, mature adipocytes, preadipocytes, fibroblasts, vascular smooth muscle cells, endothelial cells, monocytes, macrophages, and lymphocytes $(4,5)$. Stromal vascular fraction (SVF) corresponds to ADSC and describes the cells obtained immediately from adipose tissues after the digestion of collagenase $^{(6)}$. Adipose tissue is a promising source of adult mesenchymal stem cells for therapeutic applications because it is available in large amounts (100ml up to 1 liter) through liposuction and with minimal morbidity $(7)$. It is poorly antigenic, stable and low injury make them promising issues in autologus transplantation in tissue regeneration ${ }^{(8)}$. The aim of this study was to isolate and characterize the adipose derived stem cells (ASCs) from human liposuction aspirate.

\section{Materials and Methods}

\section{1-Sample preparation}

Subcutaneous fat biopsy sample (300ml) was obtained from elective liposuction procedures under spinal anaesthesia from female patient admitted to the plastic surgery department, Suez Canal Universi- ty Hospital. The raw lipoaspirate was processed according to established methodologies to obtain a stromal vascular fraction $(\mathrm{SVF})^{(9)}$.

\section{2-Isolation of mesenchymal cells}

Lipoaspiate was collected into a sterile jar and immediately transferred to the working lab., lipoaspirate was poured into a sterile bottle with an equal volume of prewarmed phosphate-buffered solution (PBS) with gentle shaking then allowed to separate based on the density. The aqueous supernatant was discarded, and the sediment were washed further for 2-3 times with PBS with addition of antibiotic solution to PBS as described by Forum et al.,2013 ${ }^{(10)}$. Collagenase solution was prepared ( $0.1 \%$ collagenase type I , $1 \%$ albumin, PBS, $2 \mathrm{mmol} / \mathrm{L} \mathrm{CaCl}_{2}$ ) and warmed in a $37^{\circ} \mathrm{C}$ water bath, then added to equal amount of lipid tissues in sealed sterile container and rocked at $37^{\circ} \mathrm{C}$ for 60 minutes in a water bath as described by Dominici et al.,2009 ${ }^{(11)}$. After digestion, enzyme activity was neutralized with DMEM containing 10\% FBS then filtered through $150 \mu$ nylon mesh to remove cell aggregates and C.T. debris. The filtrate was centrifuged twice at $1200 \mathrm{rpm}$ for 5 minutes at room temperature. The top most layers of oil, fat and collagenase solution were removed and discarded leaving behind the undisturbed stromal vascular fraction (SVF) pellet that used as a source of adipose derived mesenchymal cells ${ }^{(10)}$.

\section{3-Propagation of (ASCs)}

SVF pellet was plated into tissue culture flasks using stromal medium (DMEM, 10\% fetal bovine serum, $1 \%$ antibiotics and antimycotic) then incubated at $37^{\circ} \mathrm{C}$ in a humid atmosphere containing $5 \% \mathrm{CO}_{2}$ with regular examination under inverted microscope. Subsets of mesenchymal cells begin to adhere to the flask surface with a 
characteristic spindle shape morpholo$\mathrm{gy}^{(12)}$. After 24 hours incubation, nonadherent cells were removed, and the adherent cells were washed with DMEM $+10 \%$ fetal calf serum 2 times. Within 48 hours incubation, mesenchymal cells begin to divide rapidly and showed clumps or cell aggregates ${ }^{(13)}$. The medium was changed every 3-5 days ${ }^{(12)}$. After 10 days, the cell sheet was formed with $90 \%$ confluence. Adherent cells were detached by treatment with $0.25 \%$ trypsin-EDTA, then neutralized with FBS-containing culture media ${ }^{(14)}$.

\section{4-Phenotyping of ADSCS}

Surface-marker expression of ADSCs was evaluated by fluorescent activating cell sorting (FACS) flow cytometry using fluorescein conjugated mouse monoclonal antibodies against CD44, CD90 and CD45 (Biolegend, US) as described by Min Sun et al.,2013 ${ }^{(14)}$.

\section{Results}

I-Cultivation and characterization of human ADSCS

ADSCs were obtained from liposuction samples and cultured in tissue-culture flasks using collagenase digestion method. After digestion and centrifugation, stromal vascular fragment (SVF) was used as a source of adipose derived stem cells (ADSCS).

\section{A-Isolation of human ADSCs}

After 6 hours incubation at $370 \mathrm{C}$ and 5\% CO2, ADSCs shown to be rounded in shape with central nucleus and settled on the surface of tissue culture flasks [Figure $1(A)]$. Within 12 hours incubation, ADSCs begins to adhere to the surface and some cells begins to extend and change their rounded appearance [Figure 1(B)].After 24 hours incubation, non-adherent cells were removed and the adherent cells were washed with DMEM $+10 \%$ fetal calf serum 2 times. Adherent cells were shown to be more extended and take the spindle shape [Figure 1(C) and (D)]. Within 48 hours incubation, ADSCs cells begins to divide rapidly and showed clumps or cell aggregates [Figure $2(A)$ ]. After 72 hours incubation, ADSCs were divided rapidly and appear to fill the intercellular gaps with about $15 \%$ confluence [Figure 2 (B)]. After 4 days in culture, the cells appeared to be spindle shaped and formed symmetric colonies. The medium was changed every 3-5 days. After 7 days, the cell sheet was formed $60 \%$ confluence [Figure 2 (C)] and after 10 days, the cell sheet was formed with $90 \%$ confluence with the appearance of some rounded cells [Figure 2 (D)]. Ninety percent confluent adherent cell sheet were detached by treatment with $0.25 \%$ trypsin-EDTA, then neutralized with FBS-containing culture media then identified for mesenchymal surface markers and used for treatment of induced POF in rat model.

B-Identification of human ADSC phenotypes The cell surface phenotype of human adipose tissue-derived stem cells is quite similar to other mesenchymal stem cells (MSC). However, adipose-derived stem cells also exhibit unique characteristics distinct from those seen in MSCs, including cell marker CD44 and CD90. Absence of CD45 are used to exclude endothelial and hematopoietic cell populations. Flow cytometric analysis was used to identify ADSCs markers using fluorescenceactivated cell sorting (FACS). FACS analysis of ADSCs surface markers revealed expression of $\mathrm{CD} 44$, and CD9o markers in most of the adherent cells. Those markers were expressed in more than $95 \%$ of the population. In contrast, most adherent cells were negative for CD45 [Figure $3(A)$, (B) and (C)]. 

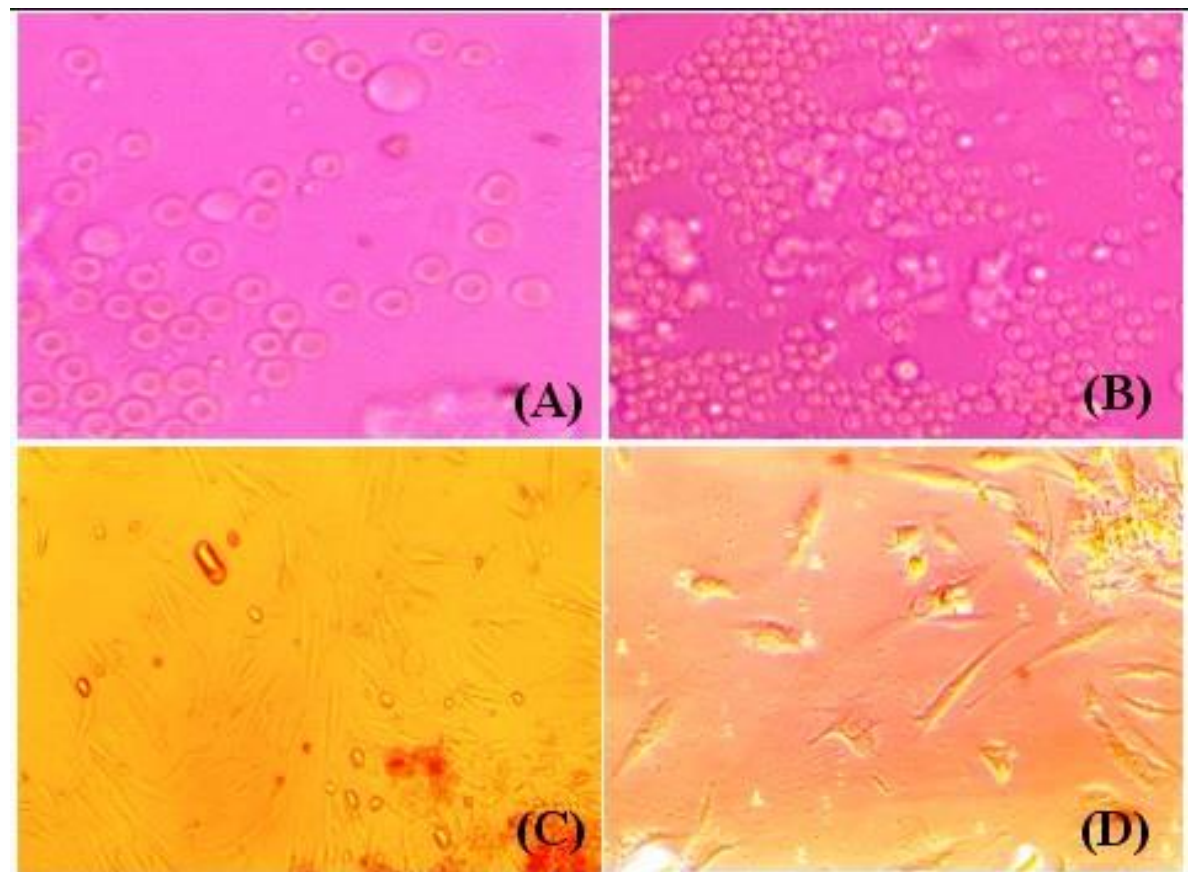

Figure (1): (A) showing ADSCs after 6 hours incubation at $370 \mathrm{C}$ and $5 \% \mathrm{CO} 2$, the cells appear as rounded cells and central nucleus (X400). (B) showing ADSCs after 12 hours incubation at $370 \mathrm{C}$ and $5 \% \mathrm{CO} 2$, the cells appear to adhere to flask surface and begins to extend and change their rounded shape (X100). (C) showing ADSCs after 24 hours incubation, cells appear more extended and take spindle shape (X100). (D) showing ADSCs after 24 hours incubation, with spindle appearance and high magnification (X400).

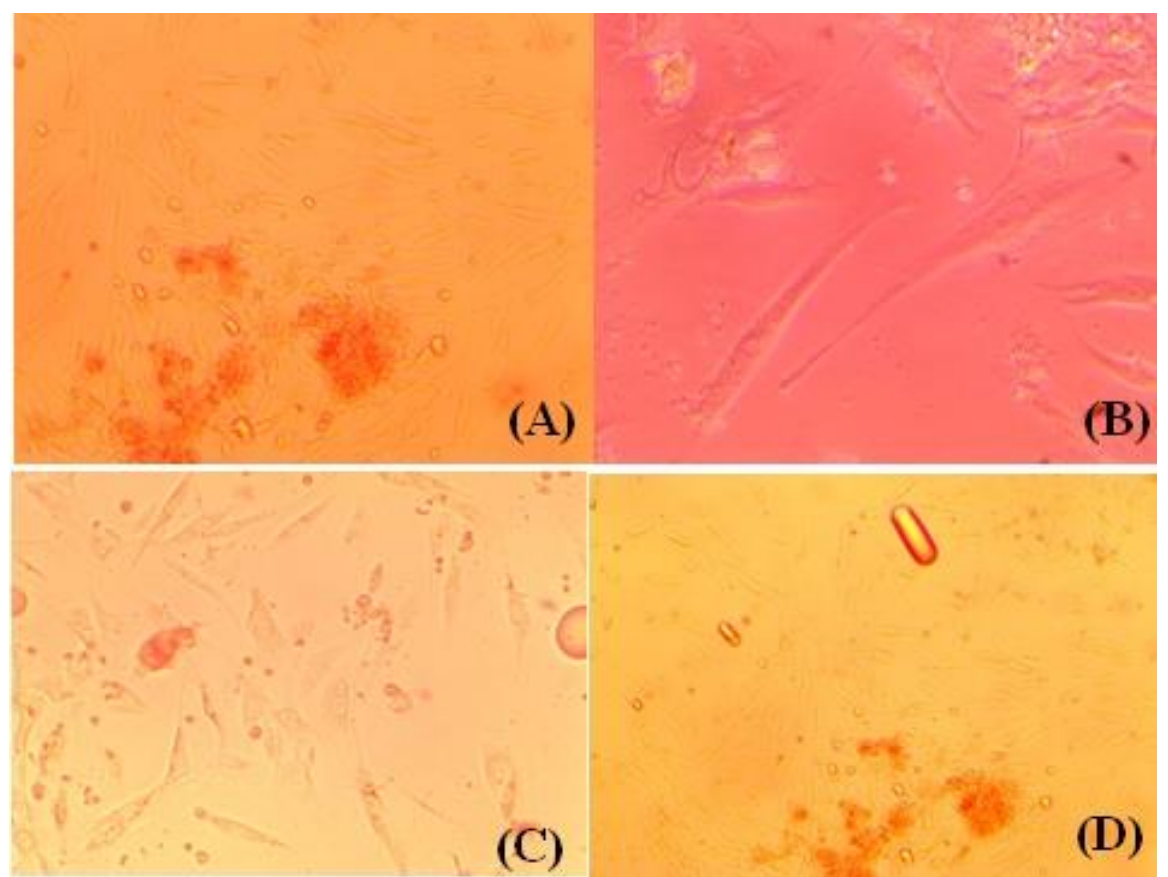

Figure (2): (A) showing ADSCs after 48 hours incubation, the cells begins to divide with formation of cell clumps and aggregates (X100). (B) showing ADSCs after 72 hours incubation, spindle mesenchymal cell appearance with 15\% confluence (X400). (C) showing ADSCs after 7 days incubation at $370 \mathrm{C}$ and $5 \% \mathrm{CO} 2$, cell sheet showed $60 \%$ confluence (X400). (D) showing ADSCs after 10 days incubation at $370 \mathrm{C}$ and $5 \% \mathrm{CO}$, cell sheet showed 90\% confluence (X100). 


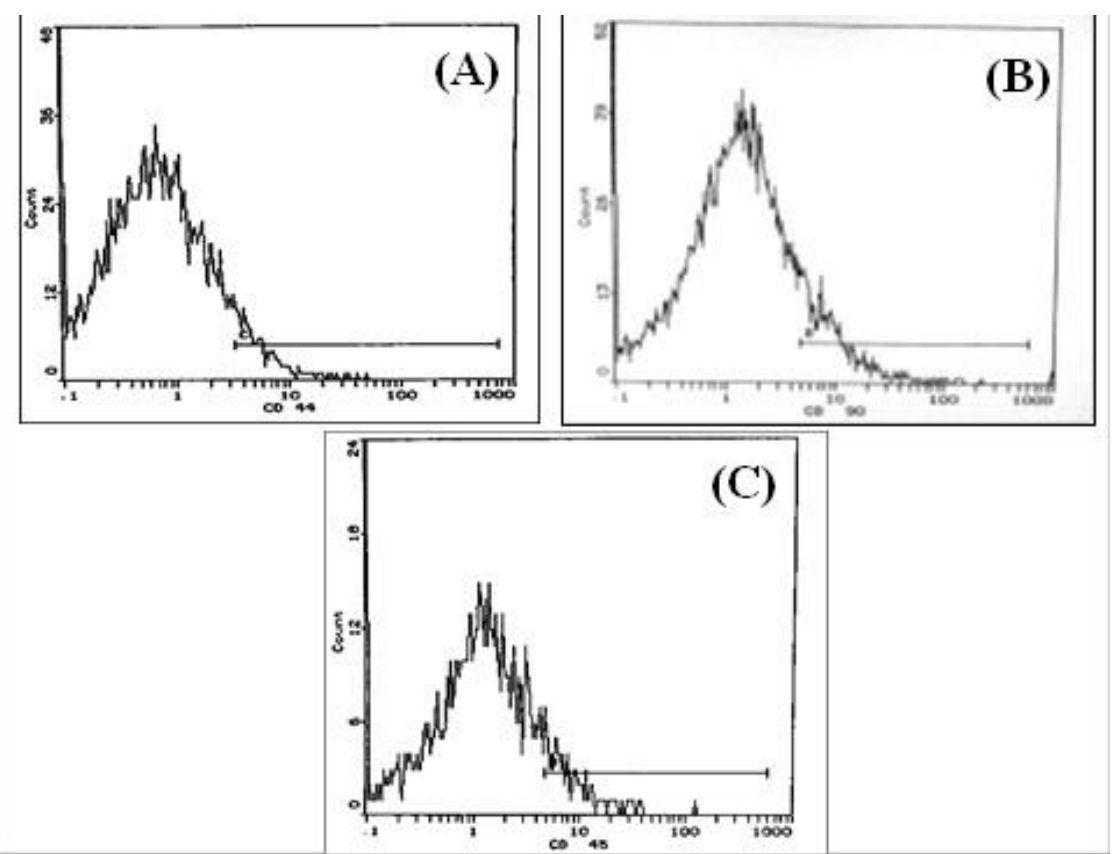

Figure (3): (A) showing positivity of ADSC for CD44 by FACS analysis (B) showing positivity of ADSC for CD9o by FACS analysis (C) showing negativity of ADSC for CD45 by FACS analysis

\section{Discussion}

ADSCs is multipotent stem cells can differentiated into multiple cell types including osteoblasts, chondrocytes, adipocytes, myocytes, vascular endothelial cells and neurons(3). ADSCs is advantageous than other stem cells as it is abundant in individuals, easily harvested from patient himself, high cellular proliferation in vitro, multipotent capacity of cell differentiation, self-renewal and little immunogenicity. The involvement of human ADSCs in this context had not been investigated so far. Stem cells have emerged as a key element in regenerative medicine therapies with a great potential to treat chronic degenerative diseases ${ }^{(15)}$. In this study, human ADSCs were isolated from liposuction aspirate. Adipose stem cells is superior than other stem cell sources because, AD$\mathrm{SC}$ is easily to obtained during liposuction surgery ${ }^{(16)}$, minimally invasive, safe for autologous transplantation and avoids ethical problems ${ }^{(17)}$. Adipose stem cells are found in any type of white adipose tissue, including subcutaneous and omental fat.
Recent researches noticed that 98-100\% of the adipose cells in the lipoaspirate are viable ${ }^{(18)}$. Human ADSCs in the current study were isolated from SVF pellet of liposuction aspirate. Cells within the SVF can adhere readily to plastic tissue culture ware and growth of these cells is evident within 2-10 days after culture. The SVF contains population of stromal cells in addition to endothelial cells, smooth muscle cells, pericytes, fibroblasts, and circulating cell types such as leucocytes, hematopoietic stem cells or endothelial progenitor cells ${ }^{(19)}$. To obtain a high ADSCs yields, collagenase digestion method is used in this study. Collagenase treatment of liposuction aspirate is the method of choice because of dissociation of tissues when compared to other methods e.g. explant culture method, combined enzyme digestion method and high trypsin concentration method ${ }^{(20)}$. Other researchers stated that the enzymatic treatment has higher cell yield but is a more invasive way of isolating cells ${ }^{(21)}$. It is necessary to replicate the adipose tissue stem cells prior to clinical application to increase their numbers. 
Cells are passaged in appropriate media for propagation and proliferation. In the current study, fetal bovine serum and DMEM media were used to promote adhesion and proliferation of ADSCs to the surface of culture vessels ${ }^{(8)}$. Other investigators recognized that animal origin reagents are avoided in human medicine. Removal of animal origin reagents provides a high level of safety for the patient and cell transplant ${ }^{(22)}$. Within 12 hours incubation, rounded ADSCs begins to adhere to the surface and begins to extend and change their rounded appearance [Figure $1(B)$ ]. Adherent cells shown to be more extended and take the spindle shape after 24 hours incubation [Figure 1 (C) and (D)]. Within 48 hours incubation, the cells begin to divide rapidly and showed clumps or cell aggregates [Figure 2 (A)] and divided rapidly to fill the intercellular gaps with about $15 \%$ confluence after 72 hours [Figure 2 (B)]. These findings were typically observed $(9,23)$. However, In vitro, ADSCs display a cell doubling time of 2 to 4 days, depending on the culture medium and passage number ${ }^{(24)}$. In our study, we found that, the cells appeared to be spindle shaped and formed symmetric colonies after 4 days incubation. After 7 days, the cell sheet was formed $60 \%$ confluence [Figure 2 (C)] and after 10 days, the cell sheet was formed with $90 \%$ confluence [Figure 2 (D)]. This information agrees with data obtained from different studies e.g. Izadpanah et al., 2006(25). Concerning to human ADSCs identification, flow cytometric analysis was used to recognize ADSCs markers using fluorescence activated cell sorting (FACS). FACS is a specialized type of flow cytometry provides a method for sorting of heterogeneous mixture of biological cells into two or more containers, one cell at a time, based upon the specific light scattering and fluorescent characteristics of each cell. It is a scientific instrument provides fast, objective and quantitative recording of fluorescent signals from individual cells as well as physical separation of cells of particular interest ${ }^{(26)}$. Identification of ADSCs as carried out by surface marker phenotyping considered a golden standard way in characterization of ADSCs. ADSCs exhibit unique characteristics distinct from those seen in MSCs, and endothelial haemopoietic cell population ${ }^{(17)}$. According to experimental report, the present study selected CD44 and CD9o stable markers are highly expressed in ADSCs(27) and CD45 has commonly been used as a surface marker for hemopoietic stem cells ${ }^{(8)}$. FACS analysis was used to identify the expression of surface markers in ADSCs. As described in [Figure 3 (A), (B) and $(C)$ ], most of the adherent cells expressed CD44 and CD90. Those markers were expressed in more than $95 \%$ of the population. In contrast, the majority of adherent cells were negative for CD45. The expression profile of surface markers for hASCs as shown in these experiments is in line with previous reports ${ }^{(28)}$ and other groups ${ }^{(6)}$.

\section{Conclusion}

Human ADSCs were isolated successfully from human lipoaspirate.

\section{References}

1. Pittenger MF, Mackay AM, Beck SC, et al. Multilineage potential of adult human mesenchymal stem cells. science. 1999 Apr 2;284(5411):143-7.

2. Lee HJ, Selesniemi K, Niikura Y, et al. Bone marrow transplantation generates immature oocytes and rescues long-term fertility in a preclinical mouse model of chemotherapy-induced premature ovarian failure. Journal of Clinical Oncology. 2007 Aug 1;25(22):3198-204.

3. Varma MJ, Breuls RG, Schouten TE, et al. Phenotypical and functional characterization of freshly isolated adipose tissue- 
derived stem cells. Stem cells and development. 2007 Feb 1;16(1):91-104.

4. Caspar-Bauguil S, Cousin B, Galinier A, et al. Adipose tissues as an ancestral immune organ: site-specific change in obesity. FEBS letters. 2005 Jul 4;579(17):3487-92.

5. Xu H, Barnes GT, Yang Q, et al. Chronic inflammation in fat plays a crucial role in the development of obesity-related insulin resistance. The Journal of clinical investigation. 2003 Dec 15;112(12):1821-30.

6. Schäffler A, \& Büchler C. Concise review: adipose tissue-derived stromal cellsbasic and clinical implications for novel cell-based therapies. Stem cells. 2007 Apr;25(4):818-27.

7. Coleman SR. Long-term survival of fat transplants: controlled demonstrations. Aesthetic plastic surgery. 1995 Sep 1;19(5):421-5.

8. Zuk PA, Zhu M, Ashjian P, et al. Human adipose tissue is a source of multipotent stem cells. Molecular biology of the cell. 2002 Dec 1;13(12):4279-95.

9. Katz AJ, Llull R, Hedrick MH, Futrell JW. Emerging approaches to the tissue engineering of fat. Clinics in plastic surgery. 1999 Oct;26(4):587-603.

10. Shah, Forum S., Xiying Wu, Marilyn Dietrich, Jennifer Rood, and Jeffrey M. Gimble. "A non-enzymatic method for isolating human adipose tissue-derived stromal stem cells." Cytotherapy 15, no. 8 (2013): 979-985.

11. Dominici M, Paolucci P, Conte P, Horwitz EM. Heterogeneity of multipotent mesenchymal stromal cells: from stromal cells to stem cells and vice versa. Transplantation. 2009 May 15;87(9S): S36-42.

12. Dawson B, \& Trapp RG. Basic \& Clinical Biostatistics (LANGE Basic Science). New York: Lange Medical Books-McGraw-Hill; 2004.

13. Woad KJ, Watkins WJ, Prendergast D, Shelling AN. The genetic basis of premature ovarian failure. Australian and New Zealand Journal of Obstetrics and Gynecology. 2006 Jun;46(3):242-4.

14. Sun M, Wang S, Li Y, Yu L, Gu F, Wang C, Yao $Y$. Adipose-derived stem cells improved mouse ovary function after chem- otherapy-induced ovary failure. Stem cell research \& therapy. 2013 Dec;4(4):80.

15. Nabhan SK, Bitencourt M, Duval $M$, et al. Fertility recovery and pregnancy after allogeneic hematopoietic stem cell transplantation in Fanconi anemia patients. haematologica. 2010 May 21: haematol2010.

16. Zuk PA, Zhu MI, Mizuno $\mathrm{H}$, et al. Multilineage cells from human adipose tissue: implications for cell-based therapies. Tissue engineering. $2001 \mathrm{Apr}$ 1;7(2):211-28.

17. Gimble JM, Katz AJ, Bunnell BA. Adiposederived stem cells for regenerative medicine. Circulation research. 2007 May 11;100(9):1249-60.

18. Shiffman MA, Mirrafati S. Fat transfer techniques: the effect of harvest and transfer methods on adipocyte viability and review of the literature. Dermatologic surgery. 2001 Sep;27(9):819-26.

19. Tholpady SS, Llull R, Ogle RC, Rubin JP, Futrell JW, Katz AJ. Adipose tissue: stem cells and beyond. Clinics in plastic surgery. 2006 Jan 1;33(1):55-62.

20. Sensebé L, \& Fleury-Cappellesso S. Biodistribution of mesenchymal stem/stromal cells in a preclinical setting. Stem cells international. 2013;2013.

21. Takehara Y, Yabuuchi A, Ezoe K, et al. The restorative effects of adipose-derived mesenchymal stem cells on damaged ovarian function. Laboratory Investigation. 2013 Feb;93(2):181.

22. Patrikoski $M$, Juntunen $M$, Boucher $S$, et al. Development of fully defined xeno-free culture system for the preparation and propagation of cell therapy-compliant human adipose stem cells. Stem cell research \& therapy. 2013 Jun;4(2):27.

23. Yeung $T$, Georges PC, Flanagan LA, et al. Effects of substrate stiffness on cell morphology, cytoskeletal structure, and adhesion. Cell motility and the cytoskeleton. 2005 Jan;60(1):24-34.

24. Mitchell JB, McIntosh K, Zvonic S, et al. Immunophenotype of human adipose-derived cells: temporal changes in stromal-associated and stem cellassociated markers. Stem cells. 2006 Feb;24(2):376-85. 
25. Izadpanah R, Trygg C, Patel B, et al. Biologic properties of mesenchymal stem cells derived from bone marrow and adipose tissue. Journal of cellular biochemistry. 2006 Dec 1;99(5):1285-97.

26. Nakagami H, Morishita R, Maeda K, Kikuchi Y, Ogihara T, Kaneda Y. Adipose tissuederived stromal cells as a novel option for regenerative cell therapy. Journal of atherosclerosis and thrombosis. 2006;13 (2): 77-81.

27. Fu XF, He YL, Xie CH, Liu W. Bone marrow mesenchymal stem cell transplantation improves ovarian function and structure in rats with chemotherapy-induced ovarian damage. Cytotherapy. 2008 Jan 1;10(4):353-63.

28. Li H, Xu Y, Fu Q, Li C. Effects of multiple agents on epithelial differentiation of rabbit adipose-derived stem cells in 3D culture. Tissue Engineering Part A. 2012 Jun 11;18(17-18):1760-70. 